\title{
Miranda
}

Revue pluridisciplinaire du monde anglophone /

Multidisciplinary peer-reviewed journal on the English-

speaking world

$17 \mid 2018$

Paysages et héritages de David Bowie

\section{Emeline Jouve, Avignon 1968 et le Living Theatre - Mémoires d'une révolution}

\section{Marielle Pelissero}

URL : http://journals.openedition.org/miranda/15007

DOI : 10.4000/miranda. 15007

ISSN : 2108-6559

\section{Éditeur}

Université Toulouse - Jean Jaurès

\section{Référence électronique}

Marielle Pelissero, «Emeline Jouve, Avignon 1968 et le Living Theatre - Mémoires d'une révolution », Miranda [En ligne], 17 | 2018, mis en ligne le 02 octobre 2018, consulté le 16 février 2021. URL : http:// journals.openedition.org/miranda/15007 ; DOI : https://doi.org/10.4000/miranda.15007

Ce document a été généré automatiquement le 16 février 2021.

\section{cc) (i) () $\Theta$}

Miranda is licensed under a Creative Commons Attribution-NonCommercial-NoDerivatives 4.0 International License. 


\title{
Emeline Jouve, Avignon 1968 et le Living Theatre - Mémoires d'une révolution
}

\author{
Marielle Pelissero
}

\section{RÉFÉRENCE}

Emeline Jouve, Avignon 1968 et le Living Theatre - Mémoires d'une révolution, éditions Deuxième Époque, 2018, 192 p., ISBN : 978-2-37769-036-7

1 L'ouvrage Avignon 1968 et le Living Theatre - Mémoires d'une révolution, d'Émeline Jouve propose une série d'entretiens réalisés au cours des années 2016 et 2017. Publié par les éditions Deuxième époque au printemps 2018, cet ouvrage offre au lecteur un ensemble de points de vue sur l'édition 1968 du Festival d'Avignon. Ces semaines avignonnaises ont marqué l'histoire culturelle française du second vingtième siècle de façon décisive : en pleine période de révolution culturelle et sociale, le Festival accueille le Living Theatre, troupe américaine de théâtre expérimental qui considère que le théâtre doit servir à «mettre en pratique, à mettre en route la révolution »1. Julian Beck, Judith Malina et leurs compagnons provoquent dans la cité des Papes un tohu-bohu à l'échelle de ce programme, dont cet ouvrage rend compte en détail.

Émeline Jouve aborde cette séquence historique à partir de trois mots clefs qui déterminent les trois parties de l'ouvrage. Une première partie, « Histoire(s) - repères historiographiques et historiques » constitue une introduction précieuse et détaillée aux points de vue nombreux et divers que le lecteur rencontrera dans les deux parties suivantes. La deuxième partie, la plus importante et la plus étayée de l'ouvrage, s'intitule «Mémoire(s). Chroniques de spect-acteurs». Elle présente les témoignages des personnes qui ont assisté à l'édition 1968 du Festival d'Avignon ainsi qu'un recueil intéressant de photographies qui viennent illustrer l'ambiance décrite au fil des 150 pages précédentes. Une troisième partie, « Héritages - récits de re-créateurs », donne 
la parole à des artistes et/ou des chercheurs qui ont travaillé - ou travaillent - à partir des événements relatifs à la présence du Living en Avignon en 1968.

Dès la «définition du projet » qui initie l'ouvrage, E. Jouve règle son objectif sur la question des tensions. On ne pourrait choisir meilleure optique pour le sujet: dès 1968, on peut lire dans la presse qu' " avec « Paradise Now ( comme pour les événements de mai), le théâtre en est arrivé au point où il faut que les choses crèvent pour repartir et recommencer $»^{2}$. Avignon 1968 est un contexte au sein duquel les tensions entre les groupes, les personnes et les valeurs ont atteint un véritable paroxysme. Aussi, il est heureux que l'auteure ait pensé ce recueil de témoignages de façon à éclairer la nature de ces tensions. Ce dessein distingue cet ouvrage et lui confère une valeur intéressante d'un point de vue historique et critique.

4 Le repérage historique et factuel introduit un point de vue sur les événements qui considère conjointement les enjeux culturels, politiques et esthétiques. À la façon d'une enquête, Emeline Jouve présente les faits avec une rigueur journalistique et chronologique qui lui permet de définir l'ensemble du territoire que chaque témoin vient ensuite cartographier suivant son point de vue subjectif, celui du réseau auquel il appartient et du contexte dans lequel il se trouvait au moment des événements. Cette structure ingénieuse permet d'une part à l'auteure d'asseoir d'entrée la valeur strictement historique et scientifique de son ouvrage, et d'autre part, elle offre au lecteur une large perspective. Étant donné qu'il dispose de cette somme de faits dans la première partie, à laquelle il peut revenir à son gré, le lecteur apprécie pleinement les échos et les écarts entre les mémoires et les faits. Par ailleurs, au fil de ces «Histoire(s)», Émeline Jouve rend compte d'une période plus longue qui situe le tumulte des quelques semaines au cours desquelles le Living a séjourné en Avignon dans des contextes plus vastes et généraux, tels que l'histoire du Festival d'Avignon, les importantes mutations de la société d'alors, ainsi que l'histoire de l'avant-garde théâtrale, en France notamment.

5 L'auteure a veillé à la diversité des points de vue rassemblés dans la partie «mémoire (s)». Le lecteur rencontre ainsi plusieurs aspects du même événement à travers les souvenirs des membres du Living Theatre et de leurs amis contestataires, des membres de l'équipe du Festival - plus ou moins proches de Vilar, des journalistes et des spectateurs. Les amateurs d'histoire et de théâtre apprécieront tout spécialement l'attention portée au point de vue de l'équipe du Théâtre des Carmes, alors dirigé par André Benedetto, qui renseigne sur la complexité des positions politiques et esthétiques, ainsi que sur les nuances qu'un récit historique rapide a tendance à négliger. Cette attention particulière conférée à la Nouvelle Compagnie d'Avignon est aussi heureuse qu'elle est nécessaire; elle est à la mesure de son importance - encore sous-estimée - dans l'histoire du théâtre français au second XXe siècle.

6 De nombreux témoignages font état de la violence des tensions et de la brutalité des échanges. Chaque partie participe de ce climat et le lecteur découvre comment les contestataires crachent sur l'équipe du Festival, au propre comme au figuré, ou comment les «bandes de droitistes » maraudent dans la nuit et poursuivent les jeunes hippies. Le cinéaste, urbaniste et écrivain Claude Eveno, alors engagé dans la contestation, résume d'une phrase cette atmosphère: "Ce qui m'est resté de la représentation aux Carmes, ce sont les hurlements, les flics... $»^{3}$. Cette effervescence s'accompagne souvent de commentaires savoureux relatifs à la libération des mœurs : 
des hippies «presque nus » se livrent à des « quasi-partouzes », des longues chevelures et des fumeurs de haschich.

7 Les témoignages relatent et mettent en lumière l'importance des conflits et des intérêts politiques locaux, qui découlent des conflits politiques nationaux et les attisent à la fois. Ainsi, les témoins font souvent mention des querelles entre les contestataires et les membres du P.C.F., ou encore de l'influence de la C.G.T. et de l'implication directe de ses membres dans les événements relatifs au Festival et au Living. De façon surprenante et intéressante, la nature extrêmement concrète et locale de ces conflits semble quelque peu placer les membres du Living à l'écart des enjeux réels. De ce point de vue, l'ouvrage témoigne de la limite des correspondances entre les cultures et les contextes français et américains au cours de cette période. Par exemple, les diverses approches de la question de l'État et de "l'argent public", mentionnée à plusieurs reprises, constituent une problématique essentielle à la compréhension de cette séquence.

On constate au fil des entretiens que l'ensemble des témoins, quel que soit leur âge, leurs convictions politiques ou artistiques en 1968, s'accorde à saluer la mémoire de Jean Vilar. La constellation de récits compose le portrait d'un grand homme qui incarne des valeurs nobles et humanistes telles que l'altruisme, l'honnêteté et la loyauté. Ceci confère à ces événements la dimension tragique qui semble bien les caractériser. En effet, Vilar semble pris dans la tourmente d'un conflit dont l'ampleur le dépasse et qu'il ne parvient pas à comprendre, malgré son intelligence et sa vertu. L'exaspération de la jeunesse est telle que les valeurs que représente Vilar, dont le lecteur contemporain apprécie la bienveillance incontestable, sont brutalement piétinées par les jeunes contestataires, qui en sont écœurés. La figure de Vilar incarne le meilleur jour de l'époque qui disparaît alors et il pâtit, en héros tragique, de la cruauté et de l'injustice des temps qui changent. Un seul individu, aussi noble et charismatique soit-il, ne peut à lui seul stopper le tumulte d'une société qui mute, même dans le territoire circonscrit du Festival d'Avignon. Comme le dit Jean-Jacques Lebel en référence à Trotsky : «c'est le mouvement qui pousse, ce n'est pas l'inverse $»^{4}$.

9 La troisième et dernière partie est plus brève, et présente le témoignage de six artistes ou chercheurs contemporains qui s'intéressent tout particulièrement au sujet. Cette approche permet au lecteur de considérer l'influence du Living Theatre dans l'histoire esthétique et théâtrale du siècle passé et de la période actuelle. On note au passage les nombreux hommages rendus à Judith Malina : l'importance relative que l'histoire du théâtre lui confère est souvent regrettée. Les témoignages de ces praticiens rendent compte des innovations du Living du point de vue de la forme, on note en particulier la mention d'un théâtre "de corps", de l'improvisation, et de la participation du public. Néanmoins, certains témoignages de la partie précédente expriment déjà l'importance de ces «Héritage(s)», et cette partie aurait peut-être mérité un plus grand développement.

10 En conclusion, l'auteure revient sur son geste méthodologique avant de proposer un écho avec la période actuelle et le cinquantenaire de 1968. L'ouvrage d'Émeline Jouve a la qualité de se concentrer moins sur les individus que sur les problématiques qui les dépassent et qu'ils incarnent ponctuellement. C'est notamment le cas de Julian Beck, qui apparaît au fil des témoignages comme un simple - voire médiocre - avatar des contradictions latentes des idéaux révolutionnaires de 1968. La polyphonie de cet ouvrage s'accorde donc parfaitement à la polyphonie qui constitue et génère un mouvement tel que celui de 1968. La multiplicité des points de vue et des paroles tissent 
les conditions d'une situation dont l'avancée semble hors de contrôle. Comme l'auteure le précise en début d'ouvrage, une telle somme de témoignages manquait dans les publications scientifiques au sujet de 1968 et du festival d'Avignon, et cet ouvrage vient combler ce vide avec précision et clarté.

\section{NOTES}

1. Jean-Jacques Lebel, Entretiens avec Julian Beck et Judith Malina, le Living Theatre, Belfond, Paris, 1969.

2. Edith Rappoport, « Paradise now : un cataclysme théâtral », France Nouvelle, 31 juillet 1968.

3. Claude Eveno in Émeline Jouve, Avignon 1968 et le Living Theatre - Mémoires d'une révolution, Éditions Deuxième époque, 2018. p.79.

4. Jean-Jacques Lebel in Émeline Jouve, Avignon 1968 et le Living Theatre - Mémoires d'une révolution, Éditions Deuxième époque, 2018. p.102.

\section{INDEX}

Mots-clés : Living Theatre, Festival d'Avignon, 1968, avant-garde, expérimentation, théâtre et politique, Jean Vilar, Julian Beck, Judith Malina, États-Unis, révolution, héritage, histoire, mémoire, Paradise Now

Keywords : Living Theater, Festival d'Avignon, 1968, avant-garde, experimentation, theater and politics, Jean Vilar, Julian Beck, Judith Malina, U.S.A., revolution, legacy, history, memory, Paradise Now

\section{AUTEURS}

\section{MARIELLE PELISSERO}

Visiting Professor

NYU

marielle.pelissero@gmail.com 